\title{
The value of a shorter-delay arterial spin labeling protocol for detecting cerebrovascular impairment
}

\author{
Caiyu Zhuang $^{1,2} \wedge$, Julien Poublanc ${ }^{1}$, Larissa Mcketton ${ }^{1}$, Lakshmikumar Venkatraghavan $^{3}$, Olivia Sobczyk ${ }^{1}$, \\ James Duffin ${ }^{3,4}$, Adrian P. Crawley ${ }^{1}$, Joseph A. Fisher ${ }^{3,4}$, Renhua Wu ${ }^{5}$, David J. Mikulis ${ }^{1}$ \\ ${ }^{1}$ Joint Department of Medical Imaging, University Health Network, Toronto, Ontario, Canada; ${ }^{2}$ Department of Medical Imaging, the First Affiliated \\ Hospital, Medical College of Shantou University, Shantou, China; ${ }^{3}$ Department of Anaesthesia, University Health Network, University of Toronto, \\ Toronto, Ontario, Canada; ${ }^{4}$ Department of Physiology, University Health Network, Toronto, Canada; ${ }^{5}$ Department of Medical Imaging, the Second \\ Affiliated Hospital, Medical College of Shantou University, Shantou, China
}

Correspondence to: Dr. David J. Mikulis. Joint Department of Medical Imaging, 3MC-431, Toronto Western Hospital, 399 Bathurst Street, Toronto, Ontario, M5T 2S8, Canada. Email: David.Mikulis@uhn.ca; Dr. Renhua Wu. Department of Medical Imaging, The Second Affiliated Hospital, Medical College of Shantou University, Shantou 515041, China. Email: rhwu@stu.edu.cn.

Background: The aim of this study was to determine the relationship between blood oxygen level dependent (BOLD) cerebrovascular reactivity (CVR) and cerebral blood flow (CBF) obtained from arterial spin labeling (ASL) using different post labeling delays (PLD).

Methods: Forty-two patients with steno-occlusive diseases and impaired CVR were divided into two groups, one scanned with a 1.5 -second (1.5-s) and the other with a 2.5-second (2.5-s) PLD ASL protocol. For all patients, a region of interest (ROI) was drawn around the CVR impairment. This affected ROI was then left-right flipped across the brain midline to obtain the control ROI. For both groups, the difference in grey matter CVR between affected and control ROI was first tested to confirm significance. The average grey matter CBF of affected and control ROIs were then compared. The same analysis method was used to compare affected and control hemispheres.

Results: In both groups of 1.5 -s and 2.5 -s PLD, CVR values in the affected ROI $(-0.049 \pm 0.055$ and $-0.042 \pm 0.074 \% / \mathrm{mmHg}$, respectively) were significantly lower compared to that in the control ROI $(0.152 \pm 0.054$ and $0.152 \pm 0.053 \% / \mathrm{mmHg}$, respectively, $\mathrm{P}<0.0001)$. In the group with the $1.5-\mathrm{s} \mathrm{PLD}, \mathrm{CBF}$ in the affected ROI $(37.62 \pm 11.37 \mathrm{~mL} / 100 \mathrm{~g} / \mathrm{min})$ was significantly lower compared to CBF in the control ROI $(44.13 \pm 11.58 \mathrm{~mL} / 100 \mathrm{~g} / \mathrm{min}, \mathrm{P}<0.05)$. However, in the group with the 2.5 -s PLD, no significant differences could be seen between CBF in the affected ROI $(40.50 \pm 14.82 \mathrm{~mL} / 100 \mathrm{~g} / \mathrm{min})$ and CBF in the control ROI $(39.68 \pm 12.49 \mathrm{~mL} / 100 \mathrm{~g} / \mathrm{min}, \mathrm{P}=0.73)$. In the hemisphere-based analysis, CBF was significantly lower in the affected side than in the control side for the group with the 1.5-s PLD $(\mathrm{P}<0.05)$ when CVR was impaired $(\mathrm{P}<0.0001)$, but not for the group with the 2.5-s PLD $(\mathrm{P}=0.49)$.

Conclusions: In conclusion, our study reveals and highlights the value of a shorter-PLD ASL protocol, which is able to reflect CVR impairment. At the same time, we offer a better understanding of the relationship between BOLD CVR and CBF obtained from ASL.

Keywords: Arterial spin labeling (ASL); blood oxygen level dependent cerebrovascular reactivity (BOLD CVR); post labeling delay (PLD)

Submitted Feb 06, 2020. Accepted for publication Sep 15, 2020.

doi: 10.21037/qims-20-148

View this article at: http://dx.doi.org/10.21037/qims-20-148

\footnotetext{
^ ORCID: 0000-0001-7310-0756.
} 


\section{Introduction}

In the past few years, there has been a widespread application of arterial spin labeling (ASL) in the assessment of neurological and psychiatric disorders (1). However, using ASL in steno-occlusive disease assessment is far more challenging due to very long arterial transit times (ATT) observed in these patients. While ATT is well defined for both young and aging participants (2), it can vary considerably with disease state (3). Accordingly, efforts have been made to prolong post-labeling delay (PLD) or apply multi-PLD to improve the measurement of cerebral blood flow (CBF) (4). Since T1-weighted magnetic resonance imaging (MRI) for blood and grey matter is similar, as long as the PLD is longer than the longest transit time, the ASL signal becomes insensitive to variations in the arterial arrival time $(5,6)$. Therefore, with a longer delay, ASL measurements have the potential to take into account the CBF from collaterals with long ATT. However, we suggest that differentiating between well-developed (short ATT) and stagnant collateral vessels (long ATT) cannot be achieved unless a dual PLD is applied (7-9). Using a shorter PLD, the $\mathrm{CBF}$ of stagnant collateral vessels may be underestimated, but this measure may be more sensitive for detecting potential ischemic areas by comparison with healthy areas because stagnant collateral vessel flow will be missed.

Cerebrovascular reactivity (CVR) measures the sensitivity of the vasculature to a vasodilatory challenge such as $\mathrm{CO}_{2}$. CVR measures may serve to guide treatment and predict outcomes $(10,11)$, with impairment of CVR in steno-occlusive patients indicating a high risk of stroke $(12,13)$. The several types of vasoactive stimuli for measuring CVR have been discussed in detail elsewhere (14). Some studies indicate that there is significant relationship between baseline ASL values and the acetazolamide (ACZ)iodine-123-N-isopropyl-p-iodoamphetamine (IMP) value acquired with single-photon emission computed tomography (SPECT) with a 2.0-s PLD $(7,15)$. In this case the baseline CBF value measured by ASL has a significant relationship with the CVR value obtained from SPECT imaging. Blood oxygen level dependent (BOLD) MRI is a primary form of functional MRI (16). Although the potential of BOLD MRI for evaluating CVR has been demonstrated in previous literature (17-19), to the best of our knowledge, the relationship between BOLD CVR and baseline CBF values obtained from ASL has not been previously investigated. However, it is not uncommon to observe lower signal intensities in ASL maps of the hemisphere where BOLD CVR is impaired compared to the contralateral side using a 1.5-s PLD ASL protocol. With a longer delay, there is enough time for the spins to perfuse the CVR impaired tissue, and as a result, the CBF values increase, but the ability of ASL to detect potential ischemic areas corresponding to areas of impaired CVR may be decreased. Therefore, in this study, as well as investigating the relationship between BOLD CVR and CBF values measured with ASL, we also examined the influence of different PLDs in the relationship between ASL CBF and BOLD CVR. Our hypothesis was that because short PLD ASL protocols discriminate against stagnant collateral vessels, and because CVR derived from step stimuli also are reduced for regions with slow responses, then their ability to detect such impaired regions should agree for short PLD ASL but not for long PLD ASL. We tested this hypothesis in patients with BOLD CVR maps that showed unilateral impairment, where one hemisphere was more severely impaired than the other hemisphere.

\section{Methods}

\section{Subject recruitment and assessment}

This study was conducted at the Toronto Western Hospital (TWH). Patients were referred from neurosurgery or stroke prevention clinics and enrolled in ongoing CVR and ASL studies. All studies were approved by the Research Ethics Board (REB) at the University Health Network and all subjects provided their informed consent. Following REB approval, images from patients at the TWH between January 2011 and July 2017 were retrospectively screened by an experienced neuroradiologist (DJM) using the following inclusion criteria: (I) BOLD CVR maps show unilateral impairment, where one hemisphere is more severely impaired than the other hemisphere; (II) a 1.5 -s or 2.5-s PLD ASL protocol, as well as a BOLD CVR sequence were performed during the same session. Patients with motion artifacts on BOLD or ASL images were excluded from the analysis. Forty-two patients with steno-occlusive diseases met those inclusion and exclusion criteria (Table 1). Patients were then assigned into two groups based on the ASL protocol applied, namely, one group with 1.5 -s PLD (age range/mean: $25-83 ; 56$ years) and the other group with 2.5 -s PLD (age range/mean: $32-74 ; 50$ years). There is no age difference between these two groups (one-way ANOVA, $\mathrm{P}>0.05)$. Patient demographic and diagnoses are listed in Tables 2,3. 
Table 1 Patient grouping

\begin{tabular}{lccccc}
\hline Group & Male & Female & Age range/mean (years) & Moyamoya disease/syndrome & Stenosis \\
\hline 1.5-second PLD group & 8 & 13 & $25-83 / 55$ & 15 & 6 \\
2.5-second PLD group & 9 & 12 & $32-74 / 51$ & 14 & 7 \\
\hline
\end{tabular}

PLD, post labeling delay.

Table 2 Patient characteristics for the long PLD ASL group

\begin{tabular}{|c|c|c|c|c|}
\hline Case No. & Gender & Age, years & Diagnosis & Angiographic findings \\
\hline 2 & Female & 48 & Moyamoya syndrome & Right ICA bifurcation stenosis, right MCA occlusion \\
\hline 3 & Male & 36 & Moyamoya disease & Left ICA bifurcation occlusion \\
\hline 4 & Male & 32 & Moyamoya disease & Bilateral ICA bifurcation stenosis \\
\hline 6 & Female & 38 & Moyamoya syndrome & Left MCA occlusion \\
\hline 7 & Female & 51 & Moyamoya disease & Right intracranial ICA and MCA stenosis \\
\hline 8 & Female & 44 & Moyamoya disease & Left MCA stenosis \\
\hline 9 & Female & 45 & Moyamoya disease & Bilateral MCA stenosis \\
\hline 12 & Male & 58 & Moyamoya disease & Left MCA occlusion \\
\hline 13 & Male & 45 & Occlusion and stenosis of bilateral carotid artery & Right MCA occlusion \\
\hline 14 & Male & 50 & Moyamoya-like & Bilateral ICA bifurcation stenosis \\
\hline 15 & Male & 57 & Stenosis & Right MCA stenosis \\
\hline 16 & Female & 74 & Moyamoya disease & Right MCA occlusion \\
\hline 17 & Female & 51 & Moyamoya disease & Left MCA occlusion \\
\hline 18 & Male & 62 & Stenosis & Left MCA occlusion \\
\hline 19 & Female & 54 & Ischemic & Right MCA stenosis \\
\hline
\end{tabular}

ICA, internal carotid artery; MCA, middle cerebral artery; ACA, anterior cerebral artery; PLD, post labeling delay; ASL, arterial spin labeling.

\section{MRI data acquisition}

All of the MRI scans were performed on a 3.0T scanner (Signa HDX platform, GE Healthcare, Milwaukee, Wisconsin, USA) at TWH using an 8-channel phased array head coil. T1-weighted anatomical images of the entire brain were acquired using a three-dimensional spoiled gradient echo pulse sequence $[1.0 \mathrm{~mm}$ thick, matrix $256 \times 256$, field of view (FOV) $22 \mathrm{~cm}$, flip angle 12, echo time
(TE) $3.06 \mathrm{~ms}$, repetition time (TR) $7.88 \mathrm{~ms}$. BOLD MR CVR data were acquired for the entire brain using a T2*weighted echo-planar gradient-echo sequence (TR 2,000 or $2,400 \mathrm{~ms}$, TE $30 \mathrm{~ms}$, flip angle $85^{\circ}$ or $70^{\circ}$, slice thickness 5.0 or $3.5 \mathrm{~mm}$, no gap, matrix $64 \times 64,255$ temporal frames) during manipulation of arterial $\mathrm{CO}_{2}$. ASL MR data were acquired for the entire brain with $3 \mathrm{D}$ pseudo-continuous ASL sequence provided by GE (46 axial slices of $4 \mathrm{~mm}$; 
Table 3 Patient characteristics for the short PLD ASL group

\begin{tabular}{|c|c|c|c|c|}
\hline Case No. & Gender & Age, years & Diagnosis & Angiographic findings \\
\hline 2 & Female & 38 & Moyamoya syndrome & Bilateral ICA bifurcation stenosis \\
\hline 3 & Female & 45 & Moyamoya disease & Right MCA occlusion \\
\hline 4 & Male & 63 & Moyamoya disease & Bilateral MCA occlusion at origin \\
\hline 6 & Female & 62 & Moyamoya disease & Left ICA bifurcation stenosis, left MCA occlusion \\
\hline 7 & Female & 79 & Stenosis & Right ICA stenosis at the origin \\
\hline 8 & Male & 80 & Stenosis & Intracranial left ICA stenosis \\
\hline 9 & Female & 57 & Stenosis & Left MCA occlusion, right intracranial ICA, MCA, and ACA stenosis \\
\hline 12 & Female & 48 & Moyamoya disease & Right ICA occlusion \\
\hline 13 & Female & 49 & Moyamoya disease & Bilateral ICA bifurcation occlusion \\
\hline 14 & Female & 43 & Moyamoya disease & Bilateral intracranial ICA stenosis \\
\hline 15 & Female & 83 & Stenosis & Right intracranial ICA stenosis \\
\hline 16 & Male & 48 & Moyamoya disease & Left ICA bifurcation occlusion \\
\hline 17 & Female & 39 & Moyamoya disease & Bilateral proximal MCA and basilar artery occlusion \\
\hline 18 & Female & 57 & Moyamoya disease & Bilateral intracranial ICA stenosis \\
\hline 19 & Male & 25 & Moyamoya disease & Bilateral intracranial ICA stenosis \\
\hline
\end{tabular}

ICA, internal carotid artery; MCA, middle cerebral artery; ACA, anterior cerebral artery; PLD, post labeling delay; ASL, arterial spin labeling.

FOV $=220 \mathrm{~mm}$; acquisition matrix $=128 \times 128,8$ spiral arms in each 3D partition; TE $=9.8 \mathrm{~ms} ; \mathrm{PLD} / \mathrm{TR}=1,525 / 4,718$ $\mathrm{ms}$ or $\mathrm{PLD} / \mathrm{TR}=2,525 / 5,413$; flip angle $155^{\circ}$; acquisition time $=4$ minutes 17 seconds). No injection was required.

\section{Vasodilatory stimulus (gas manipulation, end-tidal $\mathrm{PCO}_{2}$ and $\mathrm{PO}_{2}$ manipulation)}

In order to manipulate arterial $\mathrm{CO}_{2}$, we used an automated gas blender that adjusts the flow and gas composition to a sequential gas delivery breathing circuit (RespirAct ${ }^{\mathrm{TM}}$, Thornhill Research Inc., Toronto, Canada) as previously described $(20,21)$. This system enables independent control of the end-tidal partial pressure of carbon dioxide $\left(\mathrm{PetCO}_{2}\right)$ and end-tidal partial pressure of oxygen $\left(\mathrm{PetO}_{2}\right)$ in cooperative patients unaffected by breathing pattern and minute ventilation. The stimulus sequence protocol used in this study was as follows: $\mathrm{PetCO}_{2}$ $40 \mathrm{mmHg}$ baseline for $60 \mathrm{~s}$, a hypercapnic step change to a $\mathrm{PetCO}_{2}$ of $50 \mathrm{mmHg}$ for $90 \mathrm{~s}$, a return to baseline for $90 \mathrm{~s}$, and a second hypercapnic step to for $120 \mathrm{~s}$ with a final return to baseline. All steps were implemented while maintaining normoxia $\left(\mathrm{PetO}_{2} \sim 110 \mathrm{mmHg}\right)$. Previous work describes the $\mathrm{PetCO}_{2}$ and $\mathrm{PetO}_{2}$ sequences used during the analysis of BOLD MRI CVR in more detail $(14,22)$.

\section{Image processing}

The acquired BOLD MRI, PetCO ${ }_{2}$ and ASL data were imported into AFNI (23) software for analysis. BOLD images were slice time-corrected, and volume-registered. $\mathrm{PetCO}_{2}$ was time-shifted to the point of maximum 


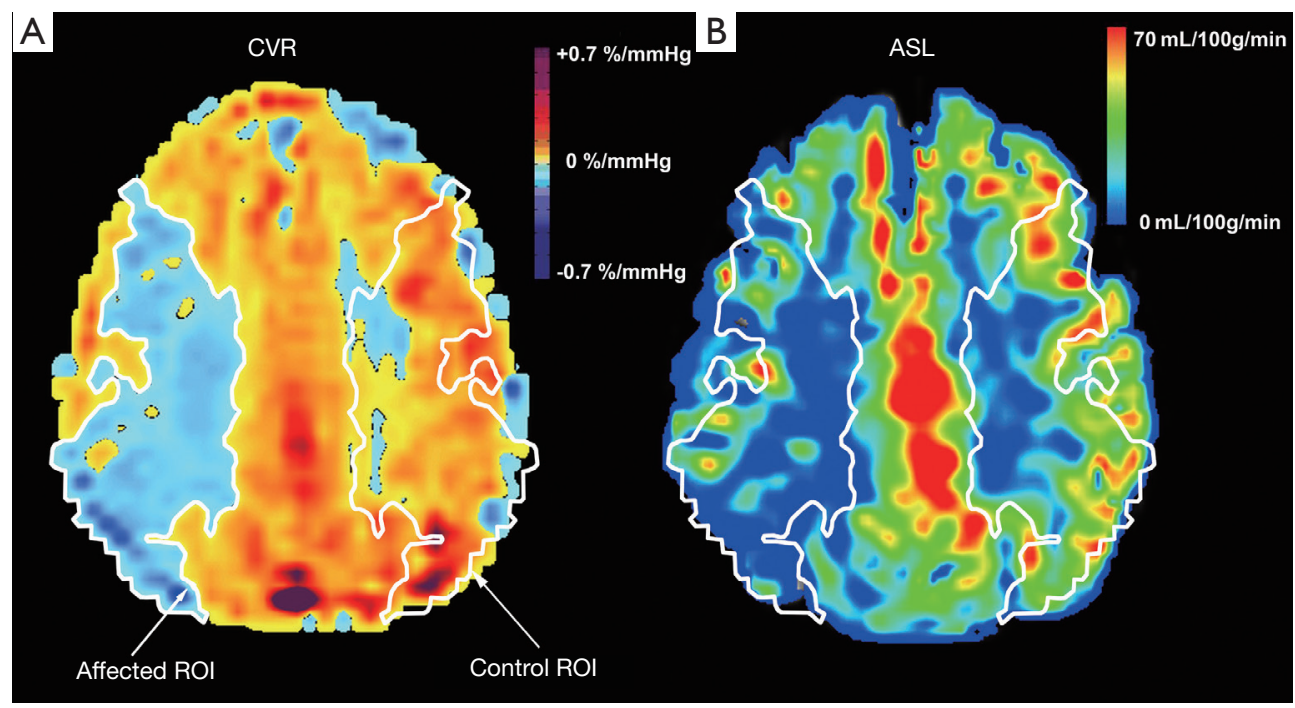

Figure 1 The region of interest (ROI) of cerebrovascular reactivity (CVR) (A) and arterial spin labeling (ASL) (B) maps for a representative subject; an 83-year-old female patient with ICA stenosis. A ROI was drawn (white line) tracing the impairment of CVR on one hemisphere, and then left/right flipped to the other hemisphere using the $\mathrm{x}$-axis in the maps as a midline. The patient's ROI in the CVR maps were then used as a mask for each subject's ASL maps.

correlation with the whole brain average BOLD signal using MATLAB software. A voxel by voxel linear regression of the BOLD signal time series against the $\mathrm{PetCO}_{2}$ data was then performed and the slope of the line of best fit was taken as the CVR measurement. CVR is therefore the change in BOLD MRI signal in response to a change in PetCO ${ }_{2}$, and expressed as $\% / \mathrm{mmHg}$. The quantification of $\mathrm{CBF}$ was available as a $\mathrm{GE}$ product (8).

\section{Data analysis}

Patients with unilateral CVR impairment were first identified by DJM. Using SPM8 (Wellcome Department of Imaging Neuroscience, Institute of Neurology, University College, London, UK), their T1-weighted anatomical images were normalized into Montreal Neurological Institute (MNI) space and segmented into cerebrospinal fluid, grey matter and white matter. Using this data, CVR and ASL grey matter masks were generated and transformed into MNI standard space.

A region of interest (ROI) was drawn around the region of CVR impairment, which was then left/right flipped across the brain midline to obtain the corresponding control ROI (Figure 1). CVR and CBF values for the impaired ROIs were compared to their respective values in the control ROIs, using a Wilcoxon matched-pairs signed ranks test, and results were considered significant if the $\mathrm{P}$ value was less than 0.05 .

In patients with long ATT collateral flow and a relatively short PLD, the image acquisition of the ASL sequence might occur before the spin tag arrives in the ROI. Therefore, a hemisphere-based analysis was also performed to validate our results. The same statistical test as above was used in this analysis.

\section{Results}

Results from the different analysis method were shown in (Table 4). CVR in the affected ROI was $-0.049 \pm 0.055 \% \mathrm{BOLD} / \mathrm{mmHg}$ for the 1.5 -s PLD group, and $-0.042 \pm 0.074 \% \mathrm{BOLD} / \mathrm{mmHg}$ for the 2.5 -s group. Those values were significantly lower compared to CVR in the control ROI, with $0.152 \pm 0.054 \% \mathrm{BOLD} / \mathrm{mmHg}$ for the 1.5 -s PLD group and $0.152 \pm 0.053 \% \mathrm{BOLD} / \mathrm{mmHg}$ for the 2.5-s PLD group $(\mathrm{P}<0.0001$, Figure $2 A, B)$.

In the group with the $1.5-\mathrm{s}$ PLD, CBF in the affected ROI $(37.62 \pm 11.37 \mathrm{~mL} / 100 \mathrm{~g} / \mathrm{min})$ was significantly lower compared to CBF in the control ROI $(44.13 \pm 11.58 \mathrm{~mL} /$ $100 \mathrm{~g} / \mathrm{min}, \mathrm{P}<0.05$, Figure $2 C$ ). However, in the group with the 2.5-s PLD, no significant differences could be seen between the CBF in the affected ROI $(40.50 \pm 14.82 \mathrm{~mL} /$ $100 \mathrm{~g} / \mathrm{min}$ ) and the CBF in the control ROI 
Table 4 Results from different analysis methods

\begin{tabular}{|c|c|c|c|}
\hline Analysis methods & Control side (mean \pm SD) & Impaired side (mean \pm SD) & $P$ value (paired $t$-test) \\
\hline BOLD CVR of 1.5 -second PLD group $(\% / \mathrm{mmHg})$ & $0.152 \pm 0.054$ & $-0.049 \pm 0.055$ & $<0.0001$ \\
\hline BOLD CVR of 2.5-second PLD group $(\% / \mathrm{mmHg})$ & $0.152 \pm 0.053$ & $-0.042 \pm 0.074$ & $<0.0001$ \\
\hline CBF of 1.5-second PLD group (mL/100 g/min) & $44.13 \pm 11.58$ & $37.62 \pm 11.37$ & $<0.05$ \\
\hline \multicolumn{4}{|l|}{ Hemisphere-based analysis } \\
\hline BOLD CVR of 1.5 -second PLD group $(\% / \mathrm{mmHg})$ & $0.211 \pm 0.060$ & $0.130 \pm 0.058$ & $<0.0001$ \\
\hline BOLD CVR of 2.5-second PLD group $(\% / \mathrm{mmHg})$ & $0.269 \pm 0.066$ & $0.179 \pm 0.068$ & $<0.0001$ \\
\hline CBF of 1.5 -second PLD group (mL/100 g/min) & $48.27 \pm 6.28$ & $45.55 \pm 7.06$ & $<0.05$ \\
\hline
\end{tabular}

ROI, region of interest; BOLD, blood oxygen dependent; CVR, cerebrovascular reactivity; CBF, cerebral blood flow; PLD, post labeling delay; SD, standard deviation.

A

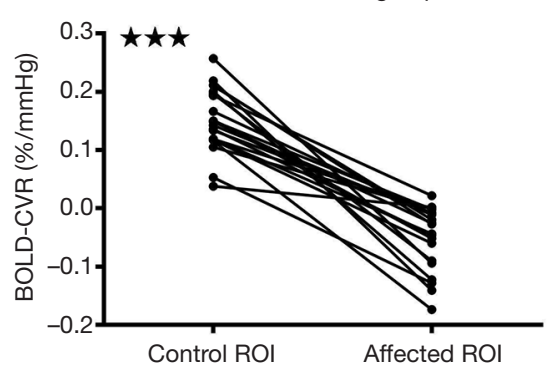

B

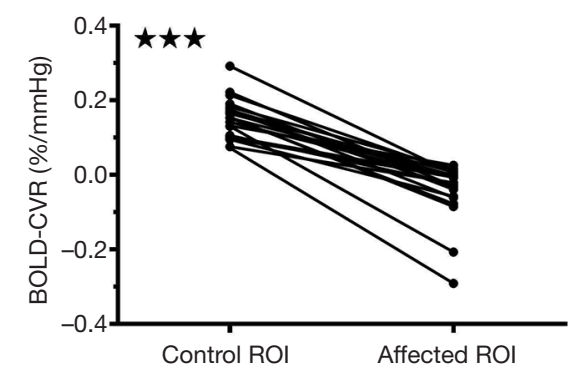

C
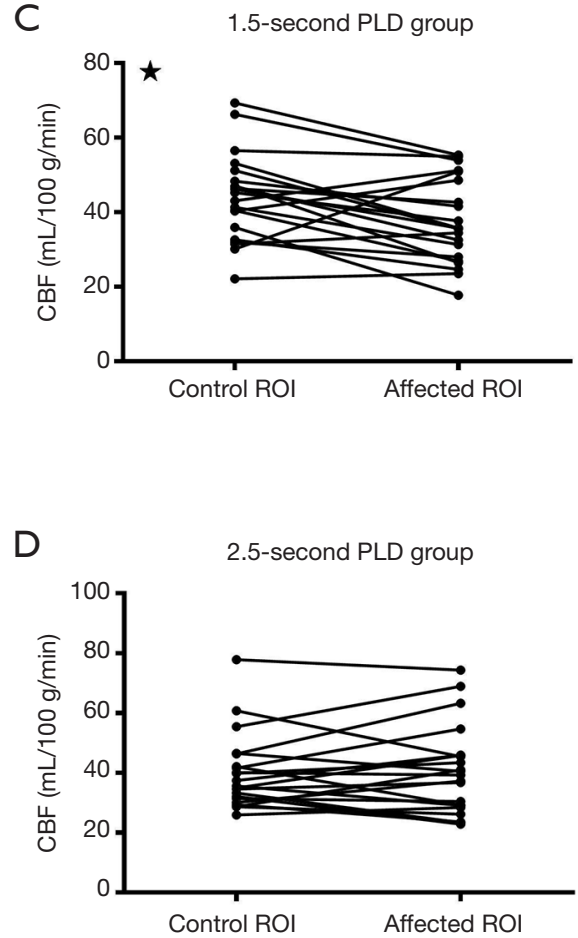

Figure 2 Figures showing the different post-labeling delays (PLDs) and their effects on cerebral blood flow (CBF) when blood oxygen level dependent cerebrovascular reactivity (BOLD CVR) was impaired, according to the region of interest (ROI)-based analysis. When BOLD CVR values were significantly decreased in the affected ROI compared with the control ROI (A,B), CBF values were significantly correspondingly decreased for the 1.5-s PLD arterial spin labeling (ASL) protocol (C). However, such a difference was not observed for the 2.5-s PLD ASL protocol (D). ${ }^{\star}, \mathrm{P}<0.05 ;{ }^{\star \star \star}, \mathrm{P}<0.0001$. 


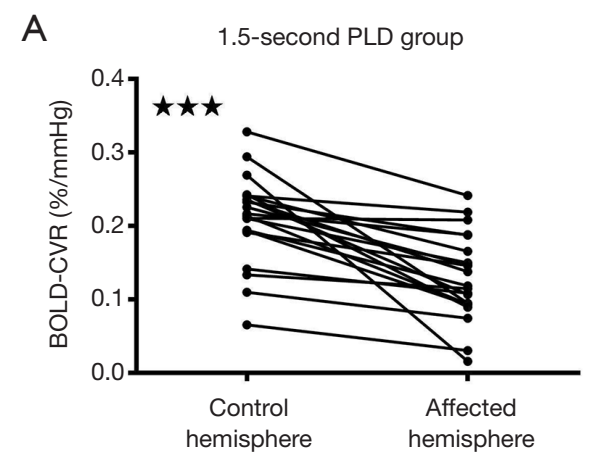

B

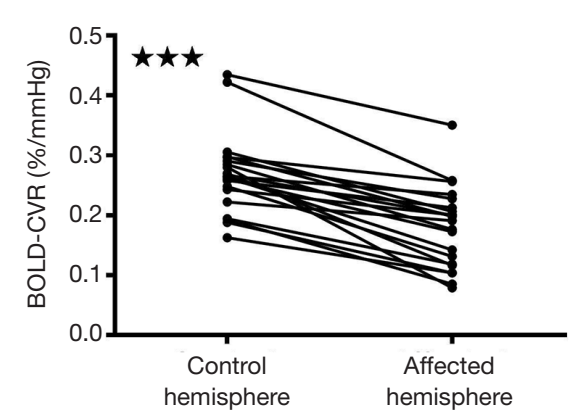

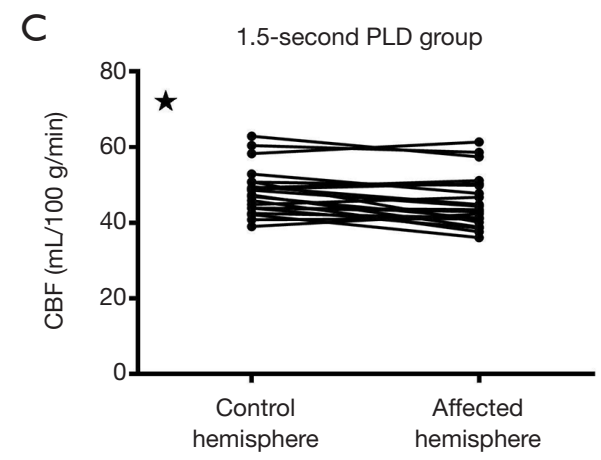

D 2.5-second PLD group

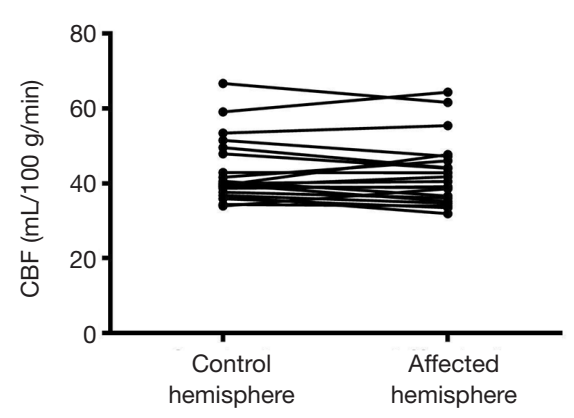

Figure 3 Figures showing different post-labeling delays (PLDs) and their effects on cerebral blood flow (CBF) when blood oxygen level dependent cerebrovascular reactivity (BOLD CVR) was impaired, according to the hemisphere-based analysis. When BOLD CVR values were significantly decreased (A,B), CBF values were significantly correspondingly decreased for the 1.5-s PLD arterial spin labeling (ASL) protocol (C), although less pronounced compared to the region of interest (ROI) analysis. Such a difference was not observed for the 2.5 -s PLD ASL protocol (D). ${ }^{\star}, \mathrm{P}<0.05 ;{ }^{\star \star \star}, \mathrm{P}<0.0001$.

\section{$(39.68 \pm 12.49 \mathrm{~mL} / 100 \mathrm{~g} / \mathrm{min}, \mathrm{P}=0.49$ ) (Figure $2 D$ ).}

Similar results were observed in the hemisphere-based analysis. In the hemisphere-based analysis, CVR in the affected hemisphere was $0.130 \pm 0.058 \% \mathrm{BOLD} / \mathrm{mmHg}$ for the 1.5 -s PLD group and $0.179 \pm 0.068 \% \mathrm{BOLD} / \mathrm{mmHg}$ for the 2.5 -s group. Those values were significantly lower compared to the CVR in the control hemisphere, with $0.211 \pm 0.060 \% \mathrm{BOLD} / \mathrm{mmHg}$ for the 1.5 -s PLD group and $0.269 \pm 0.066 \% \mathrm{BOLD} / \mathrm{mmHg}$ for the 2.5 -s PLD group $\mathrm{P}<0.0001$ (Figure $3 A, B$ ). In the group with the 1.5 -s PLD, $\mathrm{CBF}$ in the affected hemisphere $(45.55 \pm 7.06 \mathrm{~mL} / 100 \mathrm{~g} / \mathrm{min})$ was significantly lower compared to $\mathrm{CBF}$ in the control hemisphere $(48.27 \pm 6.28 \mathrm{~mL} / 100 \mathrm{~g} / \mathrm{min}, \mathrm{P}<0.05)$ (Figure $3 C$ ). However, in the group with the 2.5 -s PLD, no significant differences could be seen between the CBF in the affected hemisphere $(42.58 \pm 8.89 \mathrm{~mL} / 100 \mathrm{~g} / \mathrm{min})$ and the $\mathrm{CBF}$ in the control hemisphere $(43.10 \pm 8.58 \mathrm{~mL} / 100 \mathrm{~g} / \mathrm{min}, \mathrm{P}=0.49)$ (Figure 3D).

\section{Discussion}

Our results demonstrate that a 1.5-s PLD ASL protocol is able to show decreased CBF when BOLD CVR is impaired in support of our hypothesis, whereas the 2.5-s PLD ASL protocol is not. Nevertheless, it must be acknowledged that the relationship between regional BOLD CVR and baseline CBF is complex. The stages of cerebral hemodynamic impairment described by Powers et al. and Derdeyn et al. (24-26) suggest that CBF may be maintained until vessels exhaust their vasodilatory reserve capacity even though there may be slight decreases in CBF through the autoregulatory range. However, our results showed that with a longer PLD, the difference of CBF values acquired by ASL between the hemisphere with impaired BOLD CVR and the other hemisphere could not be observed, whereas with a shorter PLD, CBF values were significantly different. 


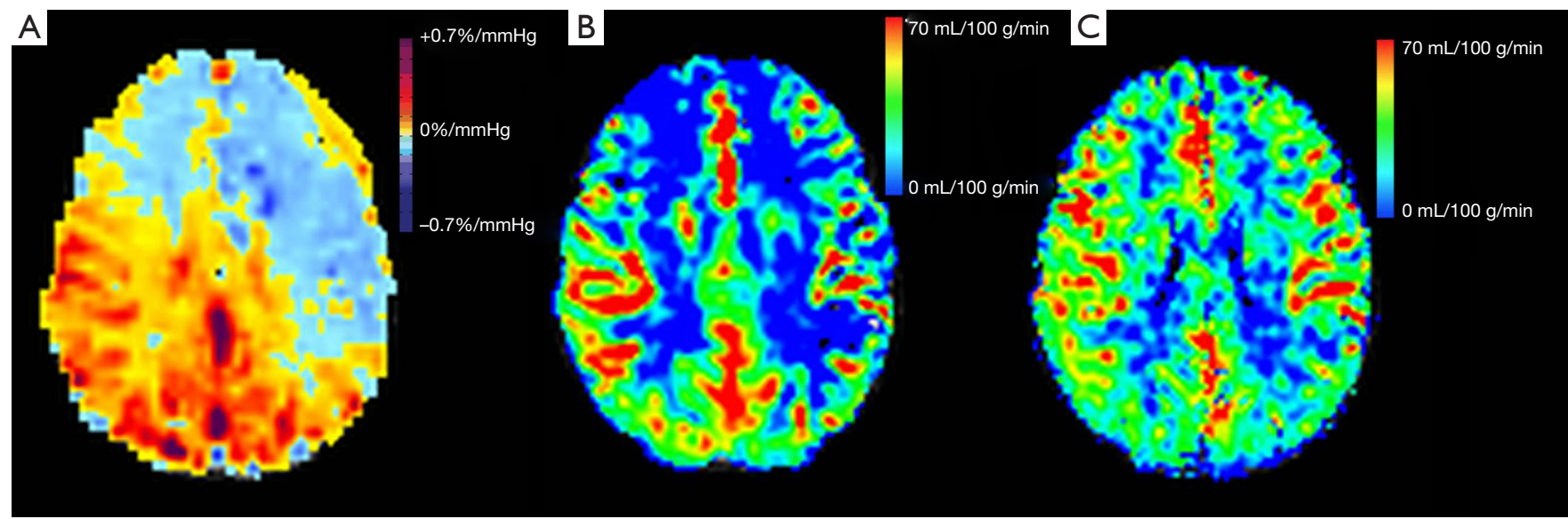

Figure 4 Figures showing cerebrovascular reactivity (CVR) (A), arterial spin labeling (ASL) with a shorter 1.5-s post-labeling delay (PLD) (B) and ASL with a longer PLD (C) for a representative subject. When the CVR was more severely impaired on the left hemisphere (A), then for the 1.5-s PLD ASL protocol, a larger area with blue color signifying lower perfusion was observed on the corresponding left side (B). However, a longer 2.5-s PLD allowed for more spins to reach the tissue, and as depicted, there was an improved perfusion in both hemispheres (C). A discrepancy in perfusion between the two hemispheres was not observed.

In Figure 4, a patient with both 1.5 -s and 2.5-s PLD ASL protocols is shown as an example. CVR was more severely impaired in the left hemisphere (Figure 4A) and the 1.5-s PLD ASL protocol correspondingly showed a larger area with low signal intensity (blue) on the left side (Figure $4 B$ ). In contrast, a longer PLD ASL protocol allowed for more spins to perfuse the tissue, resulting in an improvement of perfusion in both hemispheres (Figure 4C), so that the discrepancy in perfusion between hemispheres could not be observed. However, the serpiginous high signal intensity does indicate collateral blood flow (9), which means that although CVR is impaired, CBF is maintained by slowly responding collateral pathways $(27,28)$. Because the BOLD CVR measured by a step stimulus is reduced for slowly responding regions, it is therefore capable of detecting regions where collateral pathways are still able to maintain CBF as measured with the 2.5-s PLD ASL protocol but are slow to respond. Thus, BOLD CVR using a step stimulus enables earlier detection of potential ischemic areas compared with 2.5-s PLD ASL protocol.

Our results highlight the benefit of using an ASL sequence with a short PLD. It is well known that a shorter delay results in an underestimation of the CBF due to arterial transit time artifacts (ATA) (29), so many studies try to minimize this bias $(28,30,31)$ using longer PLD or multiple PLD ASL sequences. Not only do such ASL sequences increase scan time, but they are also not readily available on clinical scanners (8). On the other hand, we show in this study that a shorter-PLD ASL protocol is valuable since it has the potential to detect areas with a risk of ischemia that correspond to reduced CVR. In our study, we found that when CVR was decreased, the short-PLD ASL also showed hemodynamic insufficiency. Even though in the short PLD group, the CBF may be underestimated, it may serve to predict CVR impairment. Another study also tried to use ASL perfusion MRI to assess CVR (8). It compared the CBF values obtained through dual-PLD ASL protocol with CVR obtained through 123I-IMP SPECT with ACZ loading, and showed that dual PLD methods could be used to assess CVR. It indicated that in the dual-PLD methods, if CBF was improved with 2.5-s PLD compared with 1.5-s PLD, CVR would be maintained due to streaming collateral pathways, which meant only when CBF was decreased with both long and short PLD, would the CVR be impaired. However, this did not follow the stages of cerebral hemodynamic deficiency. CBF may be maintained even though CVR is impaired in the autoregulative stage, even though a slight decrease of CBF could be observed in this stage (26). Moreover, when CBF was obtained with a longer PLD, the ATA was minimized and the CBF value was more reliable. Nevertheless, it should be accepted that though short PLD ASL protocol may be sensitive in detecting CVR impairment, its specificity may be relatively low, since decreased CBF with short PLD may be due to extensive and functional collaterals, where CVR is maintained. Further study with 
A

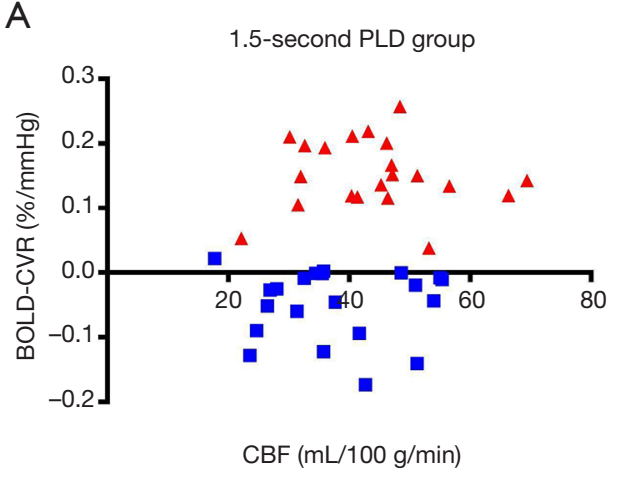

B

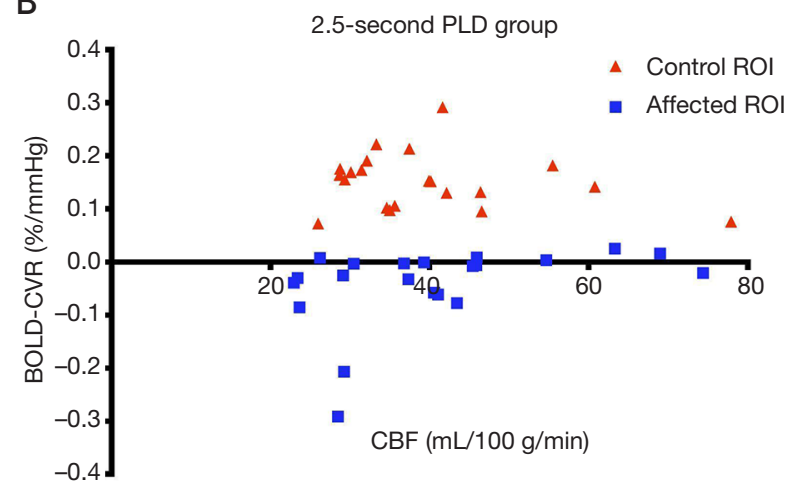

Figure 5 Relationship between blood oxygen level dependent cerebrovascular reactivity (BOLD CVR) and CBF in the 1.5-s post-labeling delay (PLD) group (A) and the 2.5-s PLD group (B). Both groups included 21 subjects.

larger patient number may be needed to know the exact sensitivity and specificity, in which patient with normal CVR should be included.

The underlying mechanism for short PLD ASL to reflect impaired CVR may be explained as follows. First, in patients with steno-occlusive disease, when CVR is exhausted (2426), their CBF may decrease, which will be presented with either short or long PLD ASL protocol. Second, the presence of collaterals does not necessarily mean that they are functional. Their adequacy can be assessed by BOLD CVR (32). Meanwhile, in patients with impaired CVR and relatively maintained true $\mathrm{CBF}, \mathrm{CBF}$ may be decreased with short PLD due to stagnant collaterals $(8,9)$. As such, short PLD ASL protocol may serve to discriminate against stagnant collateral vessels and show similar results to CVR.

It should be emphasized that in this study, we used patients' relatively unaffected hemisphere or ROI as presented in the CVR maps as a control and see if CBF acquired by short or long PLD ASL protocol was decreased in the affected hemisphere or ROI compared to the control hemisphere or ROI. The reason for us to do this are as follows. Using the contralateral hemisphere or ROI as a control will help us to get rid of the effects caused by age, since age difference contributes to a difference in $\mathrm{CBF}$ between the two groups. Second, it's hard to decide whether CBF is decreased or not since a single PLD ASL protocol could not make accurate CBF estimation due to ATA, multi-PLD ASL sequences are used for better and true CBF estimation $(28,30)$. By using the contralateral relative unaffected hemisphere or ROI as a control, we are able to decide whether CBF acquired by single PLD ASL sequence is decreased or not.

This study also demonstrates that an ASL protocols with different PLDs lead to different conclusions. For example, in contrast to other studies showing a linear relationship between CBF using a 2-s PLD ASL protocol and CVR using SPECT $(7,15)$, our results do not show such a relationship (Figure 5).

Of further interest, we found that with a 2.5-s PLD ASL protocol, in both ROI and hemisphere-based analysis, the CBF values on the affected side were higher than the control side in $10 / 21$ patients. By contrast, with the 1.5 -s PLD ASL protocol, only $5 / 21$ and $6 / 21$ patients showed higher $\mathrm{CBF}$ in the affected side than the control side (ROI and hemisphere-based analysis respectively). This finding suggests that with a longer PLD, there is a trend to overestimate CBF. Another study also showed that there is a tendency for ASL sequence to overestimate CBF, because the presence of tagged blood in arterial vessels destined to perfuse tissue in more distal regions may contaminate the ASL signal (33).

\section{Limitations}

Our study had limitations. First, this is a retrospective study. We only applied both short and long-PLD ASL to one patient. However, applying both techniques to the same patient group will help to accurately determine short or long PLD ASL's ability. Second, we use the patients' relatively unaffected hemisphere or ROI as a control, however, it's better to include patients with normal CVR and see if CBF acquired with short or long PLD ASL is normal or not. 


\section{Conclusions}

In conclusion, our study demonstrates the value of a shorter-PLD ASL protocol, which is able to reflect CVR impairment. These findings also offer a better understanding of the relationship between BOLD CVR and CBF obtained from ASL.

\section{Acknowledgments}

Funding: This work has received funding by the National Natural Science Foundation of China (Grant no. 31870981) and Grant for Key Disciplinary Project of Clinical Medicine under the Guangdong High-level University Development Program (Grant no. 002-18119101).

\section{Footnote}

Conflicts of Interest: All authors have completed the ICMJE uniform disclosure form (available at http://dx.doi. org/10.21037/qims-20-148). The authors have no conflicts of interest to declare.

Ethical Statement: The study protocol was designed in line with the guidelines outlined in the Declaration of Helsinki and approved by the Research Ethics Board (REB) at the University Health Network in Toronto. After a complete description of the study was provided to each participant, informed written consent was obtained from each participant before the study.

Open Access Statement: This is an Open Access article distributed in accordance with the Creative Commons Attribution-NonCommercial-NoDerivs 4.0 International License (CC BY-NC-ND 4.0), which permits the noncommercial replication and distribution of the article with the strict proviso that no changes or edits are made and the original work is properly cited (including links to both the formal publication through the relevant DOI and the license). See: https://creativecommons.org/licenses/by-nc$\mathrm{nd} / 4.0 /$.

\section{References}

1. Haller S, Zaharchuk G, Thomas DL, Lovblad KO, Barkhof F, Golay X. Arterial Spin Labeling Perfusion of the Brain: Emerging Clinical Applications. Radiology 2016;281:337-56.
2. Leoni RF, Oliveira IA, Pontes-Neto OM, Santos AC, Leite JP. Cerebral blood flow and vasoreactivity in aging: an arterial spin labeling study. Braz J Med Biol Res 2017;50:e5670.

3. Grade M, Hernandez Tamames JA, Pizzini FB, Achten E, Golay X, Smits M. A neuroradiologist's guide to arterial spin labeling MRI in clinical practice. Neuroradiology 2015;57:1181-202.

4. Wang DJ, Alger JR, Qiao JX, Gunther M, Pope WB, Saver JL, Salamon N, Liebeskind DS. Multi-delay multiparametric arterial spin-labeled perfusion MRI in acute ischemic stroke - Comparison with dynamic susceptibility contrast enhanced perfusion imaging. Neuroimage Clin 2013;3:1-7.

5. Alsop DC, Detre JA. Reduced transit-time sensitivity in noninvasive magnetic resonance imaging of human cerebral blood flow. J Cereb Blood Flow Metab 1996;16:1236-49.

6. Wong EC, Buxton RB, Frank LR. Quantitative imaging of perfusion using a single subtraction (QUIPSS and QUIPSS II). Magn Reson Med 1998;39:702-8.

7. Noguchi T, Kawashima M, Irie H, Ootsuka T, Nishihara M, Matsushima T, Kudo S. Arterial spin-labeling MR imaging in moyamoya disease compared with SPECT imaging. Eur J Radiol 2011;80:e557-62.

8. Haga S, Morioka T, Shimogawa T, Akiyama T, Murao K, Kanazawa Y, Sayama T, Arakawa S. Arterial Spin Labeling Perfusion Magnetic Resonance Image with Dual Postlabeling Delay: A Correlative Study with Acetazolamide Loading (123)I-Iodoamphetamine SinglePhoton Emission Computed Tomography. J Stroke Cerebrovasc Dis 2016;25:1-6.

9. Akiyama T, Morioka T, Shimogawa T, Haga S, Sayama T, Kanazawa Y, Murao K, Arakawa S. Arterial SpinLabeling Magnetic Resonance Perfusion Imaging with Dual Postlabeling Delay in Internal Carotid Artery Steno-occlusion: Validation with Digital Subtraction Angiography. J Stroke Cerebrovasc Dis 2016;25:2099-108.

10. Kuroda S, Houkin K, Ishikawa T, Nakayama N, Iwasaki Y. Novel bypass surgery for moyamoya disease using pericranial flap: its impacts on cerebral hemodynamics and long-term outcome. Neurosurgery 2010;66:1093101; discussion 1101.

11. Ogasawara K, Ogawa A, Terasaki K, Shimizu H, Tominaga T, Yoshimoto T. Use of cerebrovascular reactivity in patients with symptomatic major cerebral artery occlusion to predict 5-year outcome: comparison of xenon-133 and iodine-123-IMP single-photon 
emission computed tomography. J Cereb Blood Flow Metab 2002;22:1142-8.

12. Silvestrini M, Vernieri F, Pasqualetti P, Matteis M, Passarelli F, Troisi E, Caltagirone C. Impaired cerebral vasoreactivity and risk of stroke in patients with asymptomatic carotid artery stenosis. JAMA 2000;283:2122-7.

13. Schoof J, Lubahn W, Baeumer M, Kross R, Wallesch CW, Kozian A, Huth C, Goertler M. Impaired cerebral autoregulation distal to carotid stenosis/occlusion is associated with increased risk of stroke at cardiac surgery with cardiopulmonary bypass. J Thorac Cardiovasc Surg 2007;134:690-6.

14. Fierstra J, Sobczyk O, Battisti-Charbonney A, Mandell DM, Poublanc J, Crawley AP, Mikulis DJ, Duffin J, Fisher JA. Measuring cerebrovascular reactivity: what stimulus to use? J Physiol 2013;591:5809-21.

15. Noguchi T, Kawashima M, Nishihara M, Egashira Y, Azama S, Irie H. Noninvasive method for mapping CVR in moyamoya disease using ASL-MRI. Eur J Radiol 2015;84:1137-43.

16. Lu W, Dong K, Cui D, Jiao Q, Qiu J. Quality assurance of human functional magnetic resonance imaging: a literature review. Quant Imaging Med Surg 2019;9:1147-62.

17. Sam K, Small E, Poublanc J, Han JS, Mandell DM, Fisher JA, Crawley AP, Mikulis DJ. Reduced contralateral cerebrovascular reserve in patients with unilateral stenoocclusive disease. Cerebrovasc Dis 2014;38:94-100.

18. Smeeing DP, Hendrikse J, Petersen ET, Donahue MJ, de Vis JB. Arterial Spin Labeling and Blood Oxygen Level-Dependent MRI Cerebrovascular Reactivity in Cerebrovascular Disease: A Systematic Review and MetaAnalysis. Cerebrovasc Dis 2016;42:288-307.

19. Sam K, Poublanc J, Sobczyk O, Han JS, BattistiCharbonney A, Mandell DM, Tymianski M, Crawley AP, Fisher JA, Mikulis DJ. Assessing the effect of unilateral cerebral revascularisation on the vascular reactivity of the non-intervened hemisphere: a retrospective observational study. BMJ Open 2015;5:e006014.

20. Kisilevsky M, Hudson C, Mardimae A, Wong T, Fisher J. Concentration-dependent vasoconstrictive effect of hyperoxia on hypercarbia-dilated retinal arterioles. Microvasc Res 2008;75:263-8.

21. Ito S, Mardimae A, Han J, Duffin J, Wells G, Fedorko L, Minkovich L, Katznelson R, Meineri M, Arenovich T, Kessler C, Fisher JA. Non-invasive prospective targeting of arterial $\mathrm{P}(\mathrm{CO} 2)$ in subjects at rest. J Physiol
2008;586:3675-82.

22. Vesely A, Sasano H, Volgyesi G, Somogyi R, Tesler J, Fedorko L, Grynspan J, Crawley A, Fisher JA, Mikulis D. MRI mapping of cerebrovascular reactivity using square wave changes in end-tidal PCO2. Magn Reson Med 2001;45:1011-3.

23. Cox RW. AFNI: software for analysis and visualization of functional magnetic resonance neuroimages. Comput Biomed Res 1996;29:162-73.

24. Powers WJ. Cerebral hemodynamics in ischemic cerebrovascular disease. Ann Neurol 1991;29:231-40.

25. Powers WJ, Press GA, Grubb RL Jr, Gado M, Raichle ME. The effect of hemodynamically significant carotid artery disease on the hemodynamic status of the cerebral circulation. Ann Intern Med 1987;106:27-34.

26. Derdeyn CP, Videen TO, Yundt KD, Fritsch SM, Carpenter DA, Grubb RL, Powers WJ. Variability of cerebral blood volume and oxygen extraction: stages of cerebral haemodynamic impairment revisited. Brain 2002;125:595-607.

27. Romero JR, Pikula A, Nguyen TN, Nien YL, Norbash A, Babikian VL. Cerebral collateral circulation in carotid artery disease. Curr Cardiol Rev 2009;5:279-88.

28. Sousa I, Vilela P, Figueiredo P. Reproducibility of the quantification of arterial and tissue contributions in multiple postlabeling delay arterial spin labeling. J Magn Reson Imaging 2014;40:1453-62.

29. Amukotuwa SA, Yu C, Zaharchuk G. 3D

Pseudocontinuous arterial spin labeling in routine clinical practice: A review of clinically significant artifacts. J Magn Reson Imaging 2016;43:11-27.

30. Hara S, Tanaka Y, Ueda Y, Hayashi S, Inaji M, Ishiwata K, Ishii K, Maehara T, Nariai T. Noninvasive Evaluation of CBF and Perfusion Delay of Moyamoya Disease Using Arterial Spin-Labeling MRI with Multiple Postlabeling Delays: Comparison with (15)O-Gas PET and DSCMRI. AJNR Am J Neuroradiol 2017;38:696-702.

31. Mezue M, Segerdahl AR, Okell TW, Chappell MA, Kelly ME, Tracey I. Optimization and reliability of multiple postlabeling delay pseudo-continuous arterial spin labeling during rest and stimulus-induced functional task activation. J Cereb Blood Flow Metab 2014;34:1919-27.

32. McKetton L, Venkatraghavan L, Poublanc J, Sobczyk O, Crawley AP, Rosen C, Silver FL, Duffin J, Fisher JA, Mikulis DJ. Importance of Collateralization in Patients With Large Artery Intracranial Occlusive Disease: Long-Term Longitudinal Assessment of Cerebral Hemodynamic Function. Front Neurol 2018;9:226. 
33. Zhang K, Herzog H, Mauler J, Filss C, Okell TW, Kops ER, Tellmann L, Fischer T, Brocke B, Sturm W, Coenen $\mathrm{HH}$, Shah NJ. Comparison of cerebral blood flow

Cite this article as: Zhuang C, Poublanc J, Mcketton L, Venkatraghavan L, Sobczyk O, Duffin J, Crawley AP, Fisher JA, Wu R, Mikulis DJ. The value of a shorter-delay arterial spin labeling protocol for detecting cerebrovascular impairment. Quant Imaging Med Surg 2021;11(2):608-619. doi: 10.21037/ qims-20-148 acquired by simultaneous $[15 \mathrm{O}]$ water positron emission tomography and arterial spin labeling magnetic resonance imaging. J Cereb Blood Flow Metab 2014;34:1373-80. 